\title{
Current pharmacy practices in low- and middle-income countries; recommendations in response to the COVID-19 pandemic
}

\author{
Husnain Hamid ${ }^{1}\left[\right.$ - Rizwan Ali Masood ${ }^{2} \cdot$ Hira Tariq $^{1} \cdot$ Wahab Khalid $^{1} \cdot$ Muhammad Ateeb Rashid $^{1}$. \\ Muhammad Usman Munir ${ }^{3}$
}

Published online: 24 May 2020

(c) Springer Nature Switzerland AG 2020

\section{Introduction}

The novel coronavirus SARS-CoV-2 has spread worldwide, placing healthcare systems and healthcare professionals (HCPs) at risk. The number of countries with COVID-19, as well as the number of cases and deaths, continue to increase [1]. Healthcare systems are often unable to cope with the pandemic, with the added concern that increasing numbers of HCPs require medical care as the virus is transmitted from patient to caretaker [2].

Both private and government healthcare providers in low- and middle-income countries (LMICs) often provide low-quality patient care, even for chronic infectious diseases such as tuberculosis and malaria [4]. According to the World Health Organization, about 2 billion individuals do not have access to essential medicines [5]. In LMICs, the lack of accessibility to medications may be due to several factors such as the unaffordability and/or unavailability of drugs and legal restrictions, and may be further complicated by inappropriate medicine use, prescribing by unauthorized individuals, and poor drug quality [6]. As LMICs largely rely on imported pharmaceuticals, the effects of a pandemic and the associated lockdown conditions may result in a lack of availability of essential medicines to treat other comorbidities [7].

During public health crises such as the current COVID19 pandemic, pharmacists can provide many beneficial services, including counseling patients about methods to

Husnain Hamid

husnainhamid9@gmail.com

1 Faculty of Pharmacy, University of Central Punjab, Lahore, Pakistan

2 Department of Allied and Health Sciences, Central Queensland University, Sydney, Australia

3 College of Pharmacy, Jouf University, Sakaka, Aljouf 72388, Saudi Arabia prevent, manage, and treat the infection, as well as reporting data to governmental and other healthcare organizations. However, the quality of pharmacy practice is often poor in LMICs, even though these are the countries most in need of such practices. This commentary focuses on current pharmacy practices in LMICs and recommends innovative and emerging pharmacy services such as telepharmacy to improve outcomes and responses related to COVID-19.

\section{Pharmacy services in low- and middle-income countries}

In LMICs, patients can easily access pharmacies for advice regarding minor illnesses and childhood concerns. However, many pharmacies in LMICs function below acceptable standards and focus more on profit than on patient care [3].

The pharmacists themselves are key to improving pharmacy practice. A pharmacist must have adequate knowledge about drugs and diseases and must be vigilant in providing required services. Based on a bibliometric review, Zaheer et al. reported that the gap between pharmacy education and practice in LMICs manifested as fewer research publications and inadequate pharmacy policy and practices, especially in Africa, Eastern Europe, and Central and South America [8]. LMICs face shortcomings in pharmacy services such as the quality of practice (dispensing, labeling, and counseling) as well as the sale of antibiotics or other drugs without prescription, lack of adherence to guidelines, inadequate knowledge of severe and acute diseases, and inconsistent supply of medicines [9].

The purpose of enhancing pharmacy services is to improve the prescribing, dispensing, and appropriate utilization of drugs by patients, but such innovations are highly dependent on the overall healthcare system. As well as providing healthcare facilities and implementing policies to improve health services, developed and high-income 
countries are generally the vanguard of pharmaceutical innovations. The situation differs in LMICs, where advances in pharmaceutical and therapeutic technologies and in policies that support public health are lacking [10].

\section{Recommendations in response to the COVID-19 pandemic}

Given the generally poor pharmacy practices in LMICs, improvements in pharmaceutical services are urgently needed to fight the pandemic. The implementation and execution of innovative pharmaceutical services are also important.

LMICs often have a lack of trained pharmacists and staff to manage pharmacy services at the community level. It is highly recommended that pharmacies provide services such as patient counseling, education, home care, and psychological support, that they maintain a supply of medicines to treat and manage acute and chronic diseases, and that they have an adequate supply of personal protective equipment (e.g., masks, sanitizers, and gloves) to use to prevent the spread of COVID-19 (Fig. 1) [11]. As inappropriate, misleading, and potentially dangerous information on the prevention and management of COVID-19 may be hazardous to the community, it is vital that community pharmacists are able to provide counseling and accurate information on preventive measures and guidelines.

Innovations in pharmaceutical services relating to telepharmacy (Fig. 1) are highly accepted worldwide due to the lockdown of cities, transportation, and markets. Individuals seek healthcare advice via telephone calls with trained pharmacists, who provide counseling, education, and pharmaceutical care. These innovative pharmacy practices not only reduce the number of individuals in markets, public places, and hospitals seeking healthcare, but they also reduce nosocomial infections. Pharmacists can improve teleservices by introducing easy-to-use applications that can meet the needs of the public [12].

Hospital and clinical pharmacists have the opportunity to establish a distinct role via the implementation of pharmacovigilance services and pharmaceutical care plans for patients with comorbidities and special populations, as well as by ensuring medicines are available to hospital patients (Fig. 1). Pharmacists also need to check for drug interactions, adverse reactions, mental well-being, and minor illnesses, ensure medication safety and effectiveness, and advise the general public about preventive measures [13].

Furthermore, pharmacists can contribute during this health crisis by aiding the research and development of vaccines and evidence-based therapy (Fig. 1), thereby broadening their public role and facilitating other HCPs [14].

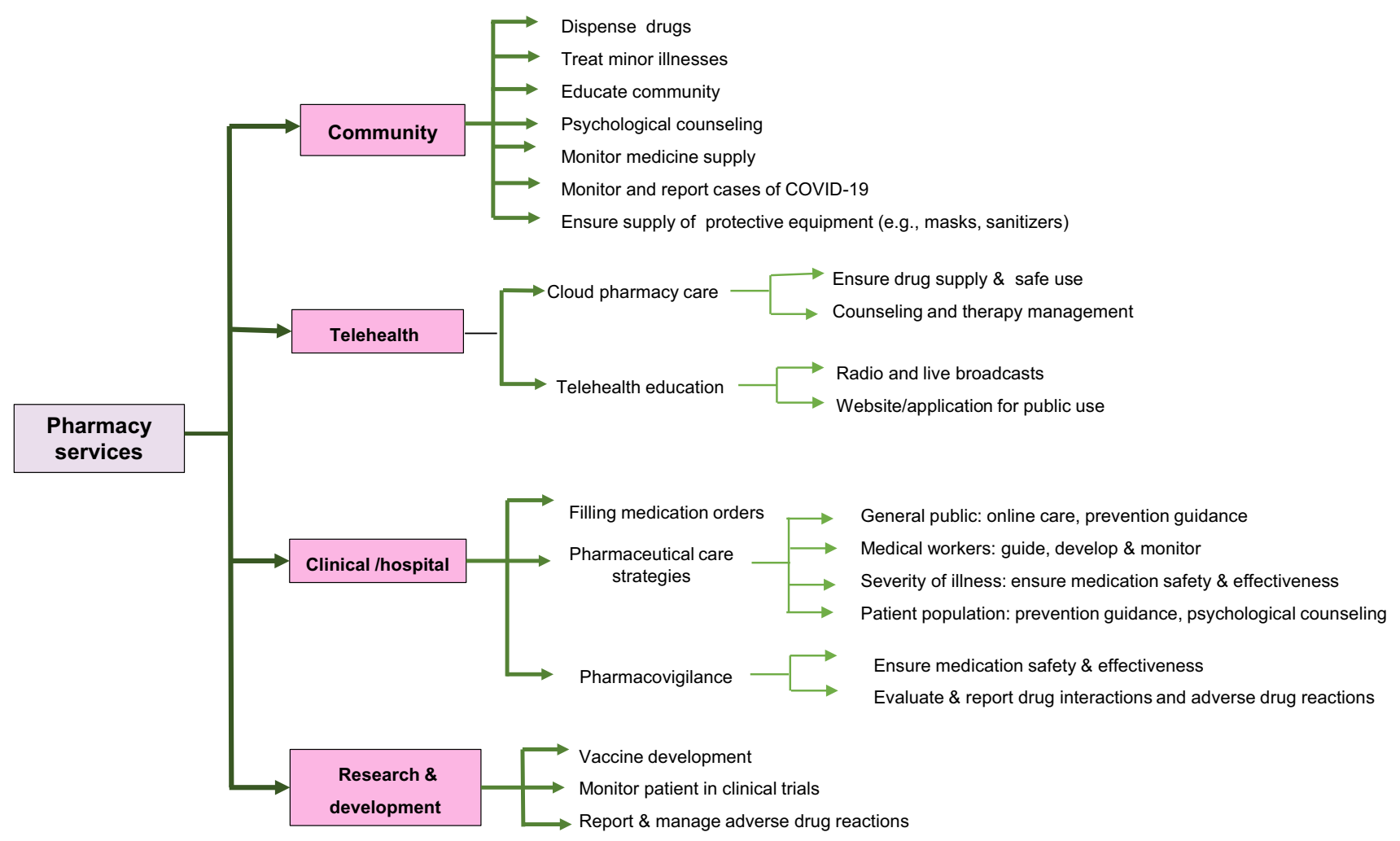

Fig. 1 Recommended pharmacy practices in response to the COVID-19 pandemic [11-13] 


\section{Conclusions}

In the scenario of a viral pandemic and public restrictions, pharmacists can provide distinct, essential services in the community, clinic, hospital, and telepharmacy settings. Measures adopted in developed countries need to be followed vigilantly in LMICs to fight against COVID-19.

\section{Take home messages}

- Foster a collaborative approach between the community and pharmacists and other HCPs to manage COVID-19.

- Enable pharmacists-an important part of the healthcare system - to play a significant role in stopping COVID-19 transmission as well as educating, managing, treating, and monitoring patients and reporting data.

- Encourage the public to consult pharmacists for their basic healthcare needs, counseling, and medications.

Acknowledgements The authors acknowledge all HCPs, especially pharmacists, who are working consistently and ceaselessly to halt this COVID-19 pandemic.

Author contributions All authors contributed equally to the conceptualization of this manuscript, the literature review, and the drafting, critical review, and writing of the manuscript.

\section{Compliance with ethical standards}

Funding None.

Conflicts of interest The authors have no conflicts of interest.

\section{References}

1. Johns Hopkins University. Coronavirus Research Center. COVID19 dashboard. https://coronavirus.jhu.edu/map.html. Accessed 28 Apr 2020.
2. Wang J, Zhou M, Liu F. Reasons for healthcare workers becoming infected with novel coronavirus disease, (COVID-19) in China. J Hosp Infect. 2020;105(1):100-1.

3. Miller R, Goodman C. Performance of retail pharmacies in lowand middle-income Asian settings: a systematic review. Health Policy Plan. 2016;31(7):940-53.

4. Beyeler N, De La YorkCruz A, Montagu D. The impact of clinical social franchising on health services in low-and middle-income countries: a systematic review. PLoS One. 2013;8(4):e60669.

5. Chan M. Ten years in public health 2007-2017. Geneva: World Health Organization; 2017.

6. Ozawa S, Shankar R, Leopold C, et al. Access to medicines through health systems in low-and middle-income countries. Kettering: Oxford University Press; 2019.

7. Kretchy IA, Asiedu-Danso M, Kretchy JP. Medication management and adherence during the COVID-19 pandemic: perspectives and experiences from LMICs. Res Soc Adm Pharm. 2020;20:30332-6.

8. Babar ZUD, Scahill SL, Akhlaq M, et al. A bibliometric review of pharmacy education literature in the context of low-to middleincome countries. Curr Pharm Teach Learn. 2013;5(3):218-32.

9. Smith F. The quality of private pharmacy services in low and middle-income countries: a systematic review. Pharm World Sci. 2009;31(3):351-61.

10. Babar ZUD, Vaughan C, Scahill S. Pharmacy practice: is the gap between the north and south widening? South Med Rev. 2012;5(1):1-2.

11. Zheng SQ, Yang L, Zhou PX, et al. Recommendations and guidance for providing pharmaceutical care services during COVID-19 pandemic: a China perspective. Res Soc Adm Pharm. 2020;20:30284-9.

12. Li H, Zheng S, Liu F, et al. Fighting against COVID-19: innovative strategies for clinical pharmacists. Res Social Adm Pharm. 2020. https://doi.org/10.1016/j.sapharm.2020.04.003

13. Ying W, Qian Y, Kun Z. Drugs supply and pharmaceutical care management practices at a designated hospital during the COVID-19 epidemic. Res Soc Adm Pharm. 2020. https://doi. org/10.1016/j.sapharm.2020.04.001 (Epub 6 Apr).

14. Hamid H, Tabassam N, Mehboob T, et al. Morbidity and mortalities by rotavirus: review for strategic measures. Pak Armed Forces Med J. 2019;69(5):1149-53. 